ORIGINAL ARTICLE

\section{Mepolizumab or Placebo for Eosinophilic Granulomatosis with Polyangiitis}

\author{
M.E. Wechsler, P. Akuthota, D. Jayne, P. Khoury, A. Klion, C.A. Langford, \\ P.A. Merkel, F. Moosig, U. Specks, M.C. Cid, R. Luqmani, J. Brown, S. Mallett, \\ R. Philipson, S.W. Yancey, J. Steinfeld, P.F. Weller, and G.J. Gleich, \\ for the EGPA Mepolizumab Study Team*
}

ABSTRACT

BACKGROUND

Eosinophilic granulomatosis with polyangiitis is an eosinophilic vasculitis. Mepolizumab, an anti-interleukin-5 monoclonal antibody, reduces blood eosinophil counts and may have value in the treatment of eosinophilic granulomatosis with polyangiitis.

\section{METHODS}

In this multicenter, double-blind, parallel-group, phase 3 trial, we randomly assigned participants with relapsing or refractory eosinophilic granulomatosis with polyangiitis who had received treatment for at least 4 weeks and were taking a stable prednisolone or prednisone dose to receive $300 \mathrm{mg}$ of mepolizumab or placebo, administered subcutaneously every 4 weeks, plus standard care, for 52 weeks. The two primary end points were the accrued weeks of remission over a 52-week period, according to categorical quantification, and the proportion of participants in remission at both week 36 and week 48. Secondary end points included the time to first relapse and the average daily glucocorticoid dose (during weeks 48 through 52). The annualized relapse rate and safety were assessed.

RESULTS

A total of 136 participants underwent randomization, with 68 participants assigned to receive mepolizumab and 68 to receive placebo. Mepolizumab treatment led to significantly more accrued weeks of remission than placebo ( $28 \%$ vs. $3 \%$ of the participants had $\geq 24$ weeks of accrued remission; odds ratio, 5.91; 95\% confidence interval [CI], 2.68 to 13.03; $\mathrm{P}<0.001$ ) and a higher percentage of participants in remission at both week 36 and week 48 (32\% vs. 3\%; odds ratio, 16.74; 95\% CI, 3.61 to 77.56; $\mathrm{P}<0.001)$. Remission did not occur in $47 \%$ of the participants in the mepolizumab group versus $81 \%$ of those in the placebo group. The annualized relapse rate was 1.14 in the mepolizumab group, as compared with 2.27 in the placebo group (rate ratio, $0.50 ; 95 \% \mathrm{CI}, 0.36$ to $0.70 ; \mathrm{P}<0.001$ ). A total of $44 \%$ of the participants in the mepolizumab group, as compared with $7 \%$ of those in the placebo group, had an average daily dose of prednisolone or prednisone of $4.0 \mathrm{mg}$ or less per day during weeks 48 through 52 (odds ratio, 0.20 ; $95 \%$ CI, 0.09 to $0.41 ; \mathrm{P}<0.001$ ). The safety profile of mepolizumab was similar to that observed in previous studies.

\section{CONCLUSIONS}

In participants with eosinophilic granulomatosis with polyangiitis, mepolizumab resulted in significantly more weeks in remission and a higher proportion of participants in remission than did placebo, thus allowing for reduced glucocorticoid use. Even so, only approximately half the participants treated with mepolizumab had protocol-defined remission. (Funded by GlaxoSmithKline and the National Institute of Allergy and Infectious Diseases; ClinicalTrials.gov number, NCT02020889.)
The authors' full names, academic degrees, and affiliations are listed in the Appendix. Address reprint requests to Dr. Wechsler at National Jewish Health, 1400 Jackson St., Denver, CO 80230, or at mikewechsler@gmail.com.

*A complete list of the members of the EGPA Mepolizumab Study Team is provided in the Supplementary Appendix, available at NEJM.org.

Drs. Weller and Gleich contributed equally to this article.

N Engl J Med 2017;376:1921-32. DOI: 10.1056/NEJMoal702079 Copyright (๑) 2017 Massachusetts Medical Society. 
$\mathrm{E}$ OSINOPHILIC GRANULOMATOSIS WITH polyangiitis (formerly known as the ChurgStrauss syndrome) is characterized by asthma, sinusitis, pulmonary infiltrates, neuropathy, and eosinophilic vasculitis of one or more endorgans..$^{1-4}$ Eosinophils are thought to induce pathogenic effects in patients with eosinophilic granulomatosis with polyangiitis by means of tissue and vascular infiltration and inflammation through a variety of mediators. ${ }^{5,6}$

Although systemic glucocorticoids form the cornerstone of treatment for eosinophilic granulomatosis with polyangiitis, ${ }^{7-9}$ most patients remain dependent on glucocorticoid therapy, and relapses are common. ${ }^{10-13}$ Furthermore, some patients do not have a sufficient response to glucocorticoids. Because recurrent relapses place the patient at risk for permanent tissue or organ damage, immunosuppressive agents are used for the induction and maintenance of remission in patients with relapsing or refractory eosinophilic granulomatosis with polyangiitis, despite a paucity of evidence supporting their efficacy in this context. ${ }^{8}$ Given the side effects that are associated with lengthy and high-dose use of glucocorticoids and immunosuppressive agents, ${ }^{14,15}$ there is a need for additional, more effective therapies.

The cytokine interleukin-5 regulates eosinophil proliferation, maturation, and differentiation and is present at increased levels in patients with eosinophilic granulomatosis with polyangiitis. ${ }^{16}$ The neutralization of interleukin-5 offers a potential therapeutic option for patients with eosinophilic granulomatosis with polyangiitis. Mepolizumab (GlaxoSmithKline) is an anti-interleukin-5 monoclonal antibody that binds to interleukin-5 and prevents its interaction with its receptor on the eosinophil surface. Mepolizumab treatment has resulted in a consistent reduction in the absolute eosinophil count, with concomitant clinical improvement in patients with other eosinophilic disorders, such as severe eosinophilic asthma. ${ }^{17-21}$ Preliminary studies have shown proof-of-concept evidence of the efficacy of interleukin-5 blockade in the treatment of patients with eosinophilic granulomatosis with polyangiitis. $22-24$ The objective of this trial was to investigate the efficacy and safety of mepolizumab versus placebo as add-on therapy in participants with relapsing or refractory eosinophilic granulomatosis with polyangiitis over a period of 52 weeks.
METHODS

\section{TRIAL DESIGN}

We conducted this randomized, placebo-controlled, double-blind, parallel-group, phase 3 trial at 31 academic centers and hospitals across nine countries (see the Supplementary Appendix, available with the full text of this article at NEJM.org). The trial was a collaboration between GlaxoSmithKline and the National Institute of Allergy and Infectious Diseases (NIAID), National Institutes of Health. NIAID funded an investigatorinitiated clinical-trial application, through which five of eight clinical sites in the United States were supported for participation in this trial. Information regarding the authors' contribution (including the contribution of authors who are employees of GlaxoSmithKline) to the trial design, data collection and analysis, and manuscript development is provided in the Supplementary Appendix. All the authors had access to the data and vouch for the accuracy and integrity of the data and analyses. Editorial support (including assistance with developing the initial draft of the manuscript, assembling tables and figures, collating authors' comments, grammatical editing, and referencing) was provided by Fishawack Indicia, funded by GlaxoSmithKline. The trial protocol is available at NEJM.org.

After screening (which lasted 1 to 4 weeks), participants were randomly assigned in a 1:1 ratio to receive subcutaneous mepolizumab at a dose of $300 \mathrm{mg}$ or matching placebo every 4 weeks, in addition to standard care (glucocorticoid treatment, with or without immunosuppressive therapy), for 52 weeks, followed by 8 weeks of follow-up (Fig. 1A, and Table S1 in the Supplementary Appendix). The glucocorticoid dose had to remain stable between baseline (randomization, which occurred at visit 2) and week 4 and could thereafter be reduced at the investigator's discretion according to a standardized recommended tapering schedule. Participants who were receiving immunosuppressive therapy were required to be taking a stable dose before baseline and for the duration of the trial.

Randomization was performed with the use of a centralized computer-generated, permutedblock schedule, stratified according to three subgroups: participation in a mechanistic-biomarker substudy in the United States, recruitment in Japan, and the remainder of recruited participants. 
Mepolizumab and placebo were prepared by staff members who were aware of the trial-group assignments but were not involved in the trial assessments. The mepolizumab and placebo preparations were identical in appearance and were administered in a blinded fashion. Clinicians who were treating and evaluating participants were unaware of the preparation of the trial agents, the trial-group assignments, and the white-cell counts and white-cell differential counts for the duration of the trial.

The trial was conducted in accordance with the ethical principles of the Declaration of Helsinki, the International Conference on Harmonisation Good Clinical Practice guidelines, and the applicable country-specific regulatory requirements. All the participants provided written informed consent.

\section{PARTICIPANTS}

Enrolled participants were at least 18 years of age, had received a diagnosis of relapsing or refractory eosinophilic granulomatosis with polyangiitis at least 6 months previously, and had been taking a stable dose of prednisolone or prednisone $(\geq 7.5$ to $\leq 50.0 \mathrm{mg}$ per day, with or without additional immunosuppressive therapy) for at least 4 weeks before the baseline visit. ${ }^{25}$ Eosinophilic granulomatosis with polyangiitis was defined as a history or presence of asthma, a blood eosinophil level of $10 \%$ or an absolute eosinophil count of more than 1000 cells per cubic millimeter, and the presence of two or more criteria that are typical of eosinophilic granulomatosis with polyangiitis (histopathological evidence of eosinophilic vasculitis, perivascular eosinophilic infiltration, or eosinophil-rich granulomatous inflammation; neuropathy; pulmonary infiltrates; sinonasal abnormality; cardiomyopathy; glomerulonephritis; alveolar hemorrhage; palpable purpura; or antineutrophil cytoplasmic antibody [ANCA] positivity, assessed at the Covance laboratory and $\mathrm{Q}^{2}$ Solutions). Participants who had granulomatosis with polyangiitis or microscopic polyangiitis at screening were excluded, as were those who had organthreatening or life-threatening eosinophilic granulomatosis with polyangiitis within 3 months before screening. Additional information regarding inclusion and exclusion criteria, stopping criteria, and definitions of relapsing and refractory disease is provided in the Supplementary Appendix.

\section{END POINTS}

The trial had two primary efficacy end points. The first primary end point was the total accrued weeks of remission. Remission was defined as a Birmingham Vasculitis Activity Score (BVAS), version 3, of 0 (on a scale from 0 to 63, with higher scores indicating greater disease activity) ${ }^{26}$ and the receipt of prednisolone or prednisone at a dose of $4.0 \mathrm{mg}$ or less per day over the 52-week period. This end point was reported as the proportions of participants who had remission for 0 weeks, for more than 0 weeks but less than 12 weeks, for at least 12 weeks but less than 24 weeks, for at least 24 weeks but less than 36 weeks, and for at least 36 weeks (categorical quantification). The second primary end point was the proportion of participants who had remission at both week 36 and week 48.

Secondary end points were the proportion of participants who had remission within the first 24 weeks and continued to have remission until week 52; the time to first relapse of eosinophilic granulomatosis with polyangiitis; and the proportions of participants with an average prednisolone or prednisone dose of $0 \mathrm{mg}$ per day, of more than $0 \mathrm{mg}$ to not more than $4.0 \mathrm{mg}$ per day, of more than $4.0 \mathrm{mg}$ to not more than $7.5 \mathrm{mg}$ per day, and of more than $7.5 \mathrm{mg}$ per day during weeks 48 through 52 . The dose level of $4.0 \mathrm{mg}$ or less per day was used as part of the remission criteria for the primary and secondary end points, so given the eligibility criteria, no participants were in remission at baseline with respect to these end points. On the basis of the European League against Rheumatism (EULAR) recommendations for clinical studies in systemic vasculitis, ${ }^{27}$ we used a second, less stringent definition of remission of a BVAS of 0 and a prednisolone or prednisone dose of $7.5 \mathrm{mg}$ or less per day for the following secondary end points: total accrued weeks of remission over the 52-week period, the proportion of participants who had remission at both week 36 and week 48, and the proportion of participants who had remission within the first 24 weeks and continued to have remission until week 52. Because the participants were required to be taking at least $7.5 \mathrm{mg}$ per day of prednisolone or prednisone before inclusion in the trial, it was possible for some of the participants to have remission at baseline with respect to these end points.

Relapse was defined as any of the following categories: active vasculitis (BVAS $>0$ ), active asth- 


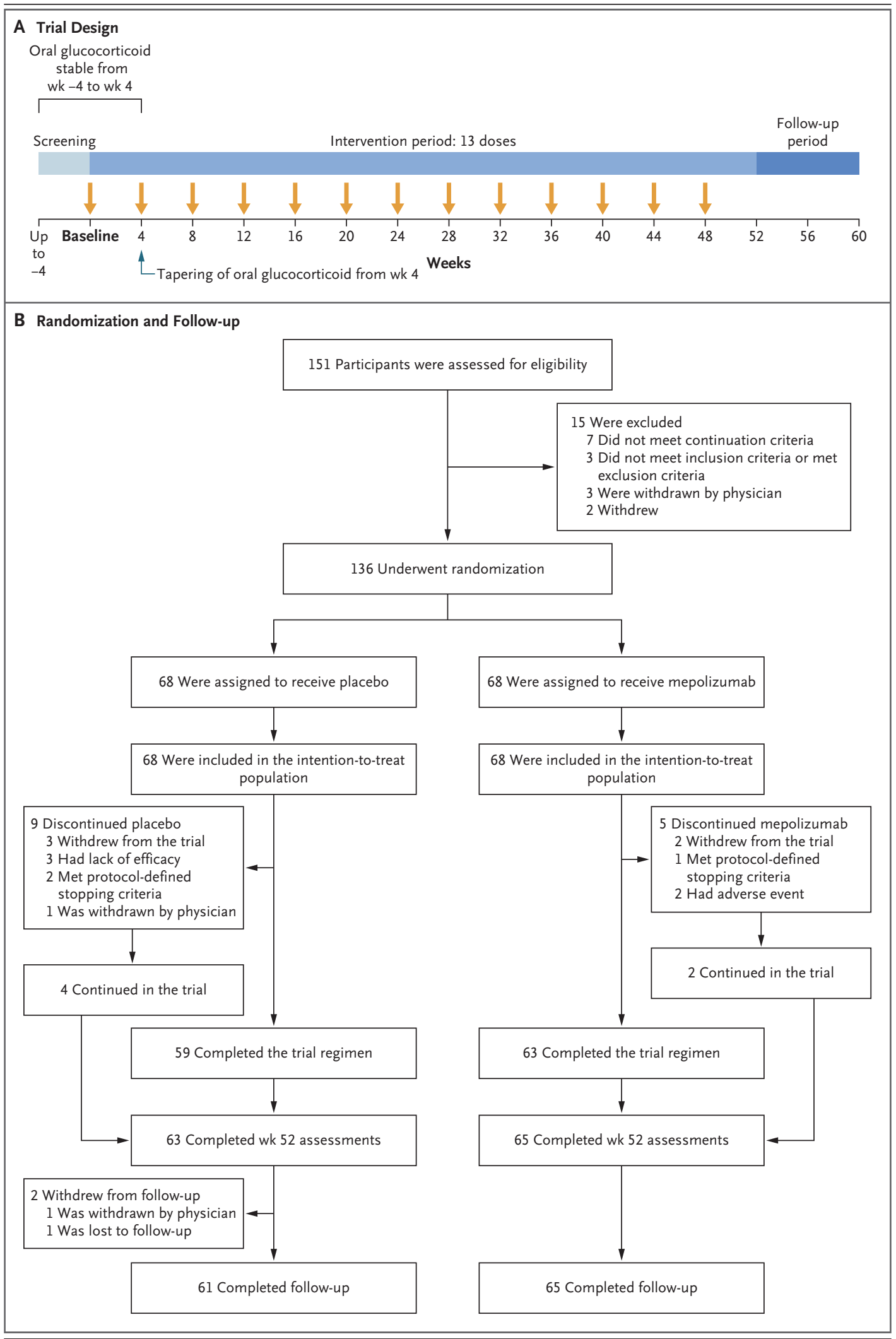

The New England Journal of Medicine

Downloaded from nejm.org at the Bodleian Libraries of the University of Oxford on June 13, 2018. For personal use only. No other uses without permission. Copyright @ 2017 Massachusetts Medical Society. All rights reserved. 
Figure 1 (facing page). Trial Design and Randomization and Follow-up of the Participants.

The continuation criteria (i.e., the criteria required for undergoing randomization) included the following: glucocorticoid and immunosuppressive therapy stability (the dose had to be stable for $\geq 4$ weeks before randomization); acceptable laboratory assessments, hepatitis status, and liver-function tests (see the Supplementary Appendix); and no clinically significant abnormality on electrocardiography. During the intervention period, participants in the mepolizumab group received $300 \mathrm{mg}$ of mepolizumab plus standard care, and those in the placebo group received matching placebo plus standard care. Mepolizumab or placebo was administered subcutaneously. The intention-to-treat population was used for the primary analysis.

ma symptoms or signs with a corresponding worsening in the score on the Asthma Control Questionnaire, version 6 (ACQ-6; range, 0 to 6 points, with higher scores indicating worse disease control; minimal clinically important difference, 0.5 points), ${ }^{28,29}$ or active nasal or sinus disease with a corresponding worsening in at least one of the sinonasal-symptom items leading to one of the following: an increase in the glucocorticoid dose to more than $4.0 \mathrm{mg}$ per day of prednisolone (or equivalent), an initiation of or increase in immunosuppressive therapy, or hospitalization. Relapses could be categorized by the local investigator as being of more than one type. Details regarding the instruments used and other prespecified end points (e.g., the change from baseline in the forced expiratory volume in 1 second, forced vital capacity, ACQ-6 score, Sino-nasal Outcome Test-22 score [range, 0 to 110 points, with higher scores indicating worse quality of life; minimal clinically important difference, 8.9 points], vascular damage index score [range, 0 to 63 points, with a score of 0 indicating no damage], and BVAS value) are provided in the Supplementary Appendix. The annualized relapse rate (i.e., the estimated rate, defined as the number of relapses per year, from a negative binomial model) was also assessed. Safety variables included adverse events and serious adverse events, including systemic and local injection-site reactions.

\section{STATISTICAL ANALYSIS}

The sample-size calculation was based on the primary end point of the total accrued weeks of remission over the 52-week period. We calculat- ed that an estimated sample of 130 participants (65 participants in each group) would provide the trial with at least $90 \%$ power to detect a significant between-group difference (at a two-sided P value of 0.05 ) of 29 percentage points in the percentage of participants who had remission for at least 24 weeks. The power calculation used a test for proportions and assumed that $25 \%$ of the participants in the placebo group would have accrued remission of at least 24 weeks, as compared with $54 \%$ of those in the mepolizumab group. These values are equivalent to an odds ratio of 3.5. To control for type I error, the two primary end points had to be positive in order for the trial to be considered successful. Per-protocol adjustment for multiple comparisons is described in the Supplementary Appendix. In practice, no adjustment was required.

Efficacy end points were assessed in the intention-to-treat population, which included all the participants who underwent randomization and received at least one dose of mepolizumab or placebo, and were analyzed according to the randomized trial groups. Safety end points were analyzed according to the actual regimen received. Ordered categorical data were analyzed with the use of proportional-odds regression. For remission, an odds ratio of more than 1 would indicate a higher proportion of participants in the higher remission-duration categories and a lower proportion of participants in the lower remissionduration categories in the mepolizumab group than in the placebo group. For the reduction in the oral glucocorticoid dose, an odds ratio of less than 1 would indicate a higher proportion of participants in the lower dose categories and a lower proportion of participants in the higher dose categories in the mepolizumab group than in the placebo group. Binary outcomes were analyzed with the use of logistic regression. Time to first relapse and time to first major relapse (defined as an organ- or life-threatening event or a BVAS $\geq 6$ or an asthma or sinonasal relapse requiring hospitalization) were analyzed with the use of Cox proportional-hazards regression. Frequency of relapse and frequency of major relapse were analyzed with the use of a negative binomial generalized linear model with a log-link function. Outcomes that were assessed at multiple time points were analyzed with the use of mixed-model, repeated-measures methods. The baseline dose of 
prednisolone or prednisone, the baseline BVAS, and geographic region (North America, Europe, or Japan) were used as covariates in the analyses specified above. Demographic characteristics (in the intention-to-treat population) and safety end points (in the as-treated population) were assessed by means of a t-test for continuous variables and Fisher's exact test for categorical variables.

Participants who discontinued mepolizumab or placebo continued to be followed until the end of the trial, when possible, and all efficacy data were included in the analysis. All the analyses were performed with the use of SAS software, version 9 (SAS Institute).

\section{RESULTS}

\section{PARTICIPANT POPULATION}

Participants were recruited from February 2014 through June 2015. Follow-up continued until September 2016. In total, 151 participants were enrolled, and 136 underwent randomization, of whom 68 were randomly assigned to receive mepolizumab and 68 to receive placebo. All the participants received at least one dose of the trial regimen and were included in the intention-to-treat population. A total of 14 participants (5 participants in the mepolizumab group and 9 in the placebo group) discontinued mepolizumab or placebo prematurely, and 10 participants ( 3 in the mepolizumab group and 7 in the placebo group) withdrew from the trial (Fig. 1B, and Table S2 in the Supplementary Appendix). The characteristics of the participants at baseline and history of eosinophilic granulomatosis with polyangiitis are shown in Table 1, and in Table S3 in the Supplementary Appendix.

\section{EFFICACY}

The trial met the two primary end points. Participants in the mepolizumab group had a significantly greater accrued time in remission over the 52-week period than did those in the placebo group: $28 \%$ of the participants in the mepolizumab group, as compared with $3 \%$ of those in the placebo group, had remission for at least 24 weeks (odds ratio, 5.91; 95\% confidence interval [CI], 2.68 to 13.03; P<0.001) (Table 2 and Fig. 2A). A total of $47 \%$ of the participants in the mepolizumab group, as compared with $81 \%$ of those in the placebo group, did not have remission, according to the definition of this primary end point. A higher percentage of participants in the mepolizumab group than in the placebo group had remission at both week 36 and week 48 (32\% vs. $3 \%$; odds ratio, 16.74 ; $95 \%$ CI, 3.61 to 77.56 ; $\mathrm{P}<0.001$ ) (Table 2). The analysis of accrued weeks of remission according to subgroup is shown in Table S4 in the Supplementary Appendix.

The efficacy with mepolizumab versus placebo among the 57 participants who had an absolute eosinophil count of less than 150 cells per cubic millimeter at baseline was limited $(21 \%$ vs. $7 \%$ of the participants had remission for $\geq 24$ weeks; odds ratio, 0.95 ; $95 \% \mathrm{CI}, 0.28$ to 3.24). By contrast, among the 79 participants with an absolute eosinophil count of 150 cells or more per cubic millimeter at baseline, there was a benefit with mepolizumab over placebo (33\% vs. $0 \%$ of the participants had remission for $\geq 24$ weeks; odds ratio, 26.10; 95\% CI, 7.02 to 97.02). More participants in the mepolizumab group than in the placebo group had remission within the first 24 weeks of the trial and remained in remission until week 52 (19\% vs. $1 \%$; odds ratio, 19.65; $95 \%$ CI, 2.30 to 167.93; $\mathrm{P}=0.007$ ) (Table 2). The remission end points that used the EULAR remission criteria (BVAS of 0 and a prednisolone or prednisone dose of $\leq 7.5 \mathrm{mg}$ per day) are shown in Figure S1 and Table S5 in the Supplementary Appendix.

The time to first relapse over the 52-week period was significantly longer in participants who received mepolizumab than in those who received placebo $(56 \%$ vs. $82 \%$ of the participants had a relapse within the 52-week period; hazard ratio, 0.32 ; $95 \%$ CI, 0.21 to $0.50 ; \mathrm{P}<0.001$ ) (Table 2 and Fig. 2B). The annualized relapse rate was $50 \%$ lower in the mepolizumab group than in the placebo group (1.14 vs. 2.27; rate ratio, $0.50 ; 95 \% \mathrm{CI}$, 0.36 to $0.70 ; \mathrm{P}<0.001$ ) (Table S6 in the Supplementary Appendix). Vasculitis relapses occurred in $43 \%$ of the participants receiving mepolizumab and in $65 \%$ of those receiving placebo; the corresponding numbers were $37 \%$ and $60 \%$ for asthma relapses and $35 \%$ and $51 \%$ for sinonasal relapses. Overall, $20 \%$ of the participants had a relapse that was classified as vasculitic only, and 54\% had a relapse that was categorized as vasculitis in combination with asthma or sinonasal relapse (Table S7 in the Supplementary Appendix). The time to the first major relapse was significantly longer in the participants in the mepolizumab group than in those in the placebo group (22\% vs. $35 \%$ of the participants had a major relapse within 


\begin{tabular}{|c|c|c|}
\hline Characteristic & $\begin{array}{l}\text { Mepolizumab } \\
\quad(\mathrm{N}=68)\end{array}$ & $\begin{array}{l}\text { Placebo } \\
(\mathrm{N}=68)\end{array}$ \\
\hline Age $-y r$ & $49 \pm 12$ & $48 \pm 14$ \\
\hline Male sex - no. (\%) & $26(38)$ & $30(44)$ \\
\hline ANCA-positive status — no. (\%) $\dagger$ & $7(10)$ & $6(9)$ \\
\hline Absolute eosinophil count per cubic millimetertr & $177 \pm 1.29$ & $172 \pm 1.35$ \\
\hline BVAS $>0$ - no. (\%)』 & $37(54)$ & $48(71)$ \\
\hline \multicolumn{3}{|l|}{ Prednisolone or prednisone dose - mg/day } \\
\hline Median & 12.0 & 11.0 \\
\hline Range & $7.5-40.0$ & $7.5-50.0$ \\
\hline Immunosuppressive therapy at baseline - no. (\%) & $41(60)$ & $31(46)$ \\
\hline \multicolumn{3}{|l|}{ EGPA diagnostic disease characteristics - no. (\%) } \\
\hline Asthma with eosinophilia & $68(100)$ & $68(100)$ \\
\hline Biopsy evidence & $25(37)$ & $31(46)$ \\
\hline Neuropathy\| & $32(47)$ & $24(35)$ \\
\hline Nonfixed pulmonary infiltrates & $50(74)$ & $48(71)$ \\
\hline Sinonasal abnormality & $64(94)$ & $64(94)$ \\
\hline Cardiomyopathy:** & $13(19)$ & $7(10)$ \\
\hline Glomerulonephritis & $1(1)$ & 0 \\
\hline Alveolar hemorrhage & $3(4)$ & $1(1)$ \\
\hline Palpable purpura & $9(13)$ & $8(12)$ \\
\hline ANCA-positive status & $13(19)$ & $13(19)$ \\
\hline Relapsing disease — no. (\%) & $51(75)$ & $49(72)$ \\
\hline Refractory disease - no. (\%) & $34(50)$ & $40(59)$ \\
\hline Duration since diagnosis of EGPA - yr & $5.2 \pm 4.4$ & $5.9 \pm 4.9$ \\
\hline Immunosuppressive therapy since diagnosis — no. (\%) & $56(82)$ & $49(72)$ \\
\hline
\end{tabular}

* Plus-minus values are means \pm SD. There were no significant between-group differences at baseline. Demographic characteristics were assessed at visit 2.

$\dagger$ Positive antineutrophil cytoplasmic antibody (ANCA) status for myeloperoxidase or proteinase 3 was assessed by means of immunoassay performed at the Covance laboratory and $\mathrm{Q}^{2}$ Solutions.

The absolute eosinophil count is presented as geometric means with standard deviation logs.

I The Birmingham Vasculitis Activity Score (BVAS) was assessed on a scale from 0 to 63, with higher scores indicating greater disease activity.

I Biopsy evidence was defined as a biopsy specimen showing histopathological evidence of eosinophilic vasculitis, perivascular eosinophilic infiltration, or eosinophil-rich granulomatous inflammation.

$\|$ Neuropathy was defined as mononeuropathy or polyneuropathy (motor deficit or nerve-conduction abnormality).

w*: The presence of cardiomyopathy was established by means of echocardiography or magnetic resonance imaging.

the 52-week period; hazard ratio, 0.51; 95\% CI, 0.26 to $0.98 ; \mathrm{P}=0.04)$. The annualized relapse rate for major relapse was $44 \%$ lower in the mepolizumab group than in the placebo group $(0.12$ vs. 0.21 ; rate ratio, $0.56 ; 95 \% \mathrm{CI}, 0.28$ to $1.14 ; \mathrm{P}=0.11$ ).

Participants who received mepolizumab had lower average doses of prednisolone or prednisone during weeks 48 through 52 than did those in the placebo group (odds ratio, 0.20; 95\% CI, 0.09 to $0.41 ; \mathrm{P}<0.001$ ) (Table S6 and Fig. S2 in the Supplementary Appendix). During weeks 48 through 52 , a total of 30 participants (44\%) receiving mepolizumab were able to taper the glucocorticoid dose to $4.0 \mathrm{mg}$ or less per day, as compared with $5(7 \%)$ receiving placebo. A total of 12 participants $(18 \%)$ receiving mepolizumab were able to discontinue prednisolone or prednisone completely, as compared with $2(3 \%)$ receiving placebo. The 


\begin{tabular}{|c|c|c|c|c|}
\hline End Point & $\begin{array}{l}\text { Mepolizumab } \\
(\mathrm{N}=68) \\
\text { no. of partic }\end{array}$ & $\begin{array}{l}\text { Placebo } \\
(\mathrm{N}=68)\end{array}$ & $\begin{array}{l}\text { Odds Ratio or } \\
\text { Hazard Ratio } \\
\text { (95\% Cl) }\end{array}$ & P Value \\
\hline \multicolumn{5}{|l|}{ Primary end points } \\
\hline Accrued weeks of remission over 52 -wk period & & & $5.91(2.68-13.03)$ & $<0.001$ \\
\hline $0 w k$ & $32(47)$ & $55(81)$ & & \\
\hline$>0$ to $<12 \mathrm{wk}$ & $8(12)$ & $8(12)$ & & \\
\hline 12 to $<24 \mathrm{wk}$ & $9(13)$ & $3(4)$ & & \\
\hline 24 to $<36 w k$ & $10(15)$ & 0 & & \\
\hline$\geq 36$ wk & $9(13)$ & $2(3)$ & & \\
\hline Remission at wk 36 and wk 48 & $22(32)$ & $2(3)$ & $16.74(3.61-77.56)$ & $<0.001$ \\
\hline \multicolumn{5}{|l|}{ Other end points } \\
\hline $\begin{array}{l}\text { Remission within the first } 24 \mathrm{wk} \text { that was sus- } \\
\text { tained until wk } 52\end{array}$ & $13(19)$ & $1(1)$ & $19.65(2.30-167.93)$ & 0.007 \\
\hline First EGPA relapse & $38(56)$ & $56(82)$ & $0.32(0.21-0.50)$ & $<0.001$ \\
\hline \multicolumn{5}{|c|}{$\begin{array}{l}\text { * Odds ratios are shown for the analyses of the two primary end points and for the secondary analysis of remission with- } \\
\text { in the first } 24 \text { weeks that was sustained until week } 52 \text {. For the analysis of accrued weeks in remission, the odds ratio is } \\
\text { for } 24 \text { or more weeks of accrued remission. Remission was defined as a BVAS of } 0 \text { (on a scale from } 0 \text { to } 63 \text {, with higher } \\
\text { scores indicating greater disease activity) and a prednisolone or prednisone dose of } 4.0 \text { mg or less per day. For the } \\
\text { time-to-event analysis of the first relapse of EPGA, the hazard ratio is shown. Participants with a first EGPA relapse } \\
\text { were those who had a relapse before the completion of the planned trial period or who withdrew prematurely from the } \\
\text { trial. }\end{array}$} \\
\hline
\end{tabular}

mean daily dose of prednisolone or prednisone, averaged over the 52-week period, was $9.2 \mathrm{mg}$ in the mepolizumab group and $13.5 \mathrm{mg}$ in the placebo group. Details regarding all the other outcomes are provided in Figures S2, S3, and S4 in the Supplementary Appendix.

\section{SAFETY}

Although there was no significant difference between the mepolizumab group and the placebo group in the percentage of participants who had an adverse event (97\% and 94\%, respectively), there was an imbalance with regard to serious adverse events during the trial period $(18 \%$ vs. $26 \%$ ) (Table 3). Some of the serious adverse events could have been related to disease activity and were therefore also considered to be relapses of eosinophilic granulomatosis with polyangiitis. Overall, the most commonly reported adverse events were headache (in 32\% of the participants in the mepolizumab group and $18 \%$ of those in the placebo group), nasopharyngitis (in 18\% and $24 \%$, respectively), arthralgia (in $22 \%$ and $18 \%$ ), sinusitis (in 21\% and 16\%), and upper respiratory tract infection (in 21\% and 16\%). Exacerbation or worsening of asthma was the most frequent serious adverse event (in 3\% of the participants in the mepolizumab group vs. $6 \%$ of those in the placebo group). Systemic reactions were infrequent and were reported with a higher incidence in the mepolizumab group than in the placebo group. The incidence of local injection-site reactions was similar in the two groups. Although cardiovascular adverse events were infrequent, one participant (a 47-year-old man) in the mepolizumab group died from cardiac arrest during the trial period; this participant had a history of coronary artery disease. None of the participants in the mepolizumab group tested positive for mepolizumab-neutralizing antibodies at any time point.

\section{DISCUSSION}

In this randomized, double-blind, placebo-controlled trial involving participants with relapsing or refractory eosinophilic granulomatosis with polyangiitis who were receiving standard-of-care therapy, mepolizumab treatment showed efficacy on the basis of the two primary end points and all secondary end points. Mepolizumab was associ- 
ated with more accrued time in remission and with a lower frequency of relapse than was placebo, which allowed for reductions in the glucocorticoid dose in the mepolizumab group. Accrued time in remission and fewer relapses are both important clinical goals, so mepolizumab fulfilled needs in this population. The adverse-event profile for mepolizumab was similar to that seen in previous studies, and no new safety signals were observed. ${ }^{17-21,23,24,30}$ Although these results are noteworthy, at the mepolizumab dose given, $47 \%$ of the participants in the mepolizumab group did not meet the primary end-point definition of remission, as compared with $81 \%$ of those in the placebo group. Although close to half the participants in the mepolizumab group had a relapse, the $50 \%$ lower rate of relapse with mepolizumab treatment than was observed with placebo in this trial was similar to the rate seen in clinical trials involving participants with other eosinophilic diseases, such as severe eosinophilic asthma. ${ }^{17-21,30}$

It remains unknown why mepolizumab was effective with regard to protocol-defined remission in approximately half the participants but not in the entire trial cohort. One consideration is that eosinophilic granulomatosis with polyangiitis is a heterogeneous disease with some manifestations being non-eosinophil driven. Another possibility is that some participants may have had long-standing, irreversible vasculitic damage that was refractory to anti-interleukin-5 therapy. Alternatively, although mepolizumab reduced blood eosinophils, the dose may have been insufficient to eliminate tissue eosinophils. Although previous open-label studies of mepolizumab involving participants with eosinophilic granulomatosis with polyangiitis examined a higher dose $(750 \mathrm{mg}$, administered intravenously every 4 weeks), it is unclear whether a higher mepolizumab dose than was used in this trial $(300 \mathrm{mg}$, administered subcutaneously) could have resulted in better responses in some participants. Finally, adrenal insufficiency that was due to long-standing glucocorticoid use may have precluded adequate glucocorticoid tapering that would result in protocol-defined remission in some participants. Although our results question the precise role of eosinophils in the pathogenesis of this complex and heterogeneous disease, ${ }^{6}$ the NIAID (grant number, U01 AI097073) is supporting further "omics" research on blood, urine, sputum, and tissue samples obtained from a subgroup of patients to address

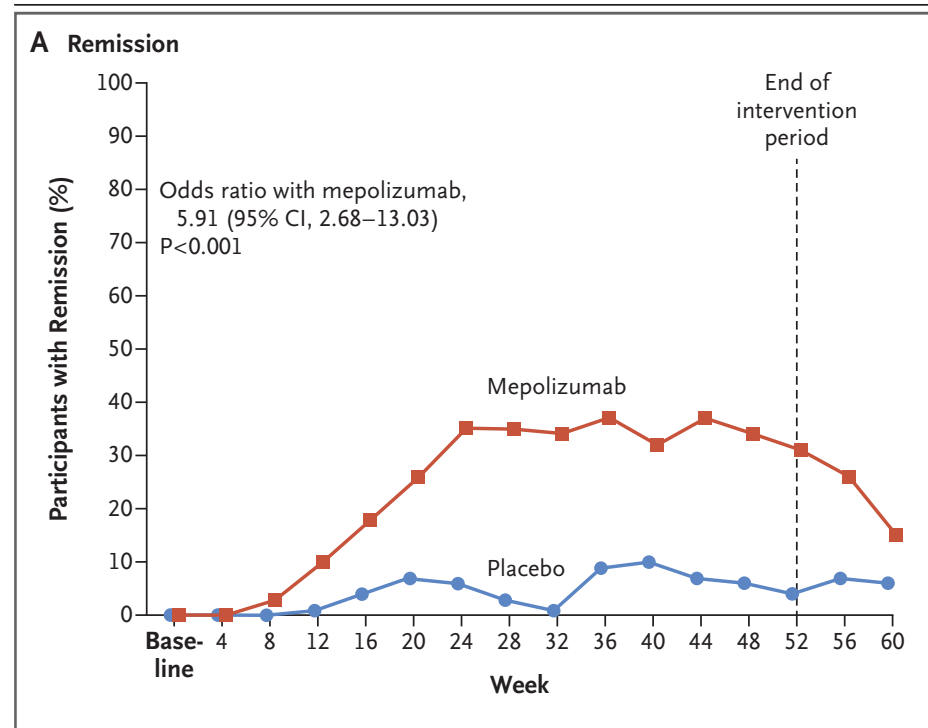

B Relapse

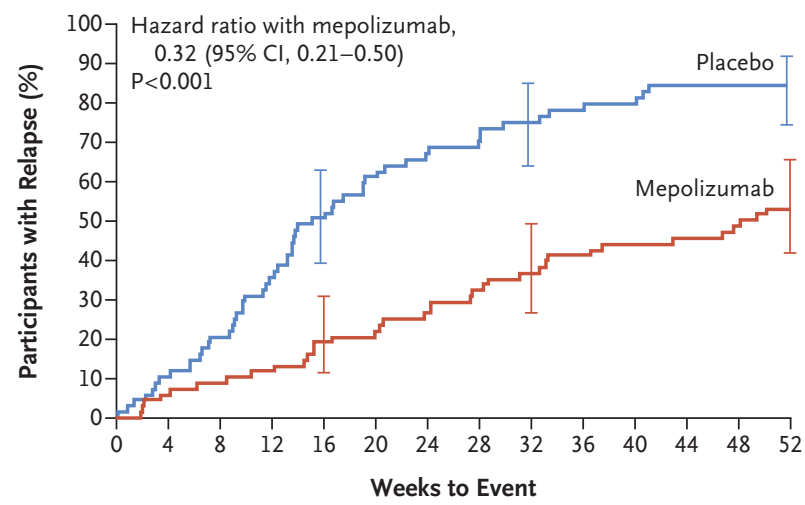

No. at Risk

Placebo $\quad 68$

Mepolizumab 68

33

55

16

43

9

Figure 2. Remission and First Relapse of Eosinophilic Granulomatosis with Polyangiitis in the Intention-to-Treat Population.

Remission was defined as a Birmingham Vasculitis Activity Score of 0 (on a scale from 0 to 63 , with higher scores indicating greater disease activity) and receipt of a prednisolone or prednisone dose of $4.0 \mathrm{mg}$ or less per day (Panel A). Data on relapse were censored at week 52 per the statistical analysis plan (Panel B). I bars at weeks 16, 32, and 52 indicate $95 \%$ confidence intervals.

questions related to disease risk and pathological features, as well as response to treatment.

The trial population was representative of patients with eosinophilic granulomatosis with polyangiitis who are treated with glucocorticoids with or without additional immunosuppressive therapy. All the participants had a history of eosinophilic asthma, and almost all had a history of sinonasal abnormality; $15 \%$ of the participants had 


\begin{tabular}{|c|c|c|}
\hline Event & $\begin{array}{l}\text { Mepolizumab } \\
\quad(\mathrm{N}=68)\end{array}$ & $\begin{array}{l}\text { Placebo } \\
(\mathrm{N}=68)\end{array}$ \\
\hline & \multicolumn{2}{|c|}{ no. of participants (\%) } \\
\hline \multicolumn{3}{|l|}{ Adverse event } \\
\hline Any event & $66(97)$ & $64(94)$ \\
\hline $\begin{array}{l}\text { Event considered by the investigator to } \\
\text { be related to the trial agent }\end{array}$ & $35(51)$ & $24(35)$ \\
\hline $\begin{array}{l}\text { Event leading to trial-agent discontinua- } \\
\text { tion or trial withdrawal }\end{array}$ & $2(3)$ & $1(1)$ \\
\hline Death & $1(1) \dagger$ & 0 \\
\hline \multicolumn{3}{|l|}{ Serious adverse eventtr } \\
\hline Any event & $12(18)$ & $18(26)$ \\
\hline $\begin{array}{l}\text { Event considered by the investigator to } \\
\text { be related to the trial agent }\end{array}$ & $3(4)$ & $3(4)$ \\
\hline \multicolumn{3}{|l|}{ Systemic or local-site reaction $\mathbb{\int}$} \\
\hline Systemic reaction & $4(6)$ & $1(1)$ \\
\hline Local-site reaction & $10(15)$ & $9(13)$ \\
\hline $\begin{array}{l}\text { Anaphylaxis considered by the investiga- } \\
\text { tor to be related to the trial agent }\end{array}$ & 0 & 0 \\
\hline \multicolumn{3}{|l|}{ Cardiovascular adverse event $\emptyset$} \\
\hline Arrhythmia & $2(3)$ & $3(4)$ \\
\hline Stroke or TIA & $1(1)$ & 0 \\
\hline Congestive heart failure & 0 & $1(1)$ \\
\hline Myocardial infarction or unstable angina & $1(1)$ & $1(1)$ \\
\hline
\end{tabular}

* There were no significant between-group differences. TIA denotes transient ischemic attack.

$\uparrow$ The event (cardiac arrest) was not considered by the physician to be related to the trial regimen.

$t$ Serious adverse events were defined as any untoward medical occurrence that resulted in death, was life-threatening, resulted in hospitalization or prolongation of existing hospitalization, resulted in disability or incapacity, was a congenital anomaly or birth defect, or was indicative of possible drug-induced liver injury with hyperbilirubinemia.

$\int$ Systemic or local-site reactions were identified by means of an electronic casereport form that was designed for the collection of data on systemic reactions.

I Cardiovascular adverse events were identified by means of an electronic casereport form that was designed for the collection of data on cardiovascular events. a history of cardiomyopathy, $41 \%$ had a history of active neuropathy, and 19\% were ANCA-positive at some time in the course of the disease. Furthermore, at baseline, the majority of participants had a BVAS score of more than 0 and the mean vascular damage index score was 4.6, which showed notable levels of vasculitic activity and considerable accrued damage. The nature of the relapses that were observed also reflects the natural course of eosinophilic granulomatosis with polyangiitis, with just $20 \%$ of the participants having a relapse that was classified as vasculitic only but $54 \%$ having relapses that were categorized as vasculitic in combination with asthma or sinonasal relapse.

The findings reflect the strengths of the trial, including a large number of enrolled participants with this rare condition, the expertise of the participating centers, and the comprehensive approach to documenting glucocorticoid use throughout the trial. Such documentation is often difficult in studies regarding the tapering of glucocorticoid doses.

This trial also had limitations. First, the BVAS, ${ }^{31-34}$ which is a tool that was developed for the assessment of vasculitis, was used to characterize disease activity and outcomes because no standard assessment tool is validated specifically for eosinophilic granulomatosis with polyangiitis. Second, the glucocorticoid dose at trial entry was varied, because participants entered the trial while they were taking a dose that had been prescribed by their own physician. However, any effect on the glucocorticoid dose that was observed in the final 4 weeks of the trial should have been mitigated, because tapering occurred with investigators who were unaware of the trial-group assignments. Third, because participants were receiving glucocorticoid therapy at trial entry, it is likely that their inflammatory markers were suppressed, which leaves little opportunity for further reduction with mepolizumab treatment. Fourth, because less than $10 \%$ of the participants were ANCApositive at baseline, analyses of outcomes according to ANCA status were not performed.

The results of our trial show an advance for patients with this rare disease. Approximately half the participants with relapsing or refractory eosinophilic granulomatosis with polyangiitis who were treated with mepolizumab had clinically relevant improvements in the rates of protocoldefined remission and relapse, which allowed for reduced glucocorticoid use. Although $81 \%$ of the participants in the placebo group had no accrued weeks of remission, $47 \%$ of those in the mepolizumab group did not have protocol-defined remission, either, which suggests that not all patients will have the same benefit.

Supported by grants from GlaxoSmithKline (115921) and the National Institute of Allergy and Infectious Diseases (NIAID), National Institutes of Health (U01 AI097073), and by the Division of Intramural Research, NIAID, National Institutes of Health.

Dr. Wechsler reports receiving consulting fees from AstraZeneca, Boston Scientific, Novartis, Vectura Group, Sunovion, Regeneron, Ambit BioScience, Meda Pharmaceuticals, Mylan, GliaCure, Tunitas Therapeutics, Genentech, Theravance Bio- 
pharma, Neurotronics, and Boehringer Ingelheim, grant support and consulting fees from Sanofi, fees for serving on a data and safety monitoring board from Sentien Biotechnologies, and consulting fees and fees for serving on an advisory board from Teva Pharmaceutical Industries; Dr. Akuthota, receiving consulting fees from Ambrx and travel fees paid by AstraZeneca; Dr. Langford, receiving grant support from Bristol-Myers Squibb and Genentech and serving as an unpaid consultant for BristolMyers Squibb; Dr. Merkel, receiving grant support and consulting fees from Bristol-Myers Squibb, ChemoCentryx, and Genentech/Roche, grant support from Celgene and MedImmune/ AstraZeneca, and consulting fees from Boston Pharmaceuticals, GlaxoSmithKline, InflaRx, and Seattle Genetics; Dr. Moosig, receiving grant support and consulting fees from Roche and consulting fees from Chugai Pharmaceuticals, Eli Lilly, and Glaxo-
SmithKline; Dr. Cid, receiving consulting fees from GlaxoSmithKline, Novartis, Roche, and Boehringer Ingelheim; Dr. Luqmani, receiving grant support from Roche and consulting fees from Roche and GlaxoSmithKline; Dr. Brown, Mr. Mallett, Mr. Yancey, and Dr. Steinfeld, being an employee of and owning stock in GlaxoSmithKline; Dr. Philipson, being an employee of Trizell; and Dr. Gleich, receiving consulting fees from Genentech. No other potential conflict of interest relevant to this article was reported.

Disclosure forms provided by the authors are available with the full text of this article at NEJM.org.

We thank the patients who participated in the trial; the trial staff; Elizabeth Juniper, M.C.S.P., M.Sc., for permission to use the Asthma Control Questionnaire; and Elizabeth Hutchinson, Ph.D., C.M.P.P., of Fishawack Indicia, for editorial support, funded by GlaxoSmithKline.

APPENDIX

The authors' full names and academic degrees are as follows: Michael E. Wechsler, M.D., Praveen Akuthota, M.D., David Jayne, F.Med. Sci., Paneez Khoury, M.D., M.H.S., Amy Klion, M.D., Carol A. Langford, M.D., M.H.S., Peter A. Merkel, M.D., M.P.H., Frank Moosig, M.D., Ulrich Specks, M.D., Maria C. Cid, M.D., Raashid Luqmani, D.M., Judith Brown, Ph.D., Stephen Mallett, M.Sc., Richard Philipson, M.D., Steve W. Yancey, M.Sc., Jonathan Steinfeld, M.D., Peter F. Weller, M.D., and Gerald J. Gleich, M.D.

The authors' affiliations are as follows: Department of Medicine, National Jewish Health, Denver (M.E.W.); the Division of Pulmonary, Critical Care, and Sleep Medicine, University of California San Diego, La Jolla (P.A.); Beth Israel Deaconess Medical Center, Boston (P.A., P.F.W.); the Department of Medicine, University of Cambridge, Cambridge (D.J.), the Department of Orthopaedics, Rheumatology, and Musculoskeletal Sciences, Botnar Research Centre, University of Oxford, Oxford (R.L.), Research and Development, ImmunoInflammation Therapy Area Unit (J.B.), and Research and Development, Statistics, Programming, and Data Standards (S.M.), GlaxoSmithKline, Uxbridge, and Trizell, Oxford (R.P.) - all in the United Kingdom; the Human Eosinophil Section, Laboratory of Parasitic Diseases, National Institute of Allergy and Infectious Diseases, National Institutes of Health, Bethesda, MD (P.K., A.K.); the Center for Vasculitis Care and Research, Cleveland Clinic, Cleveland (C.A.L.); the Division of Rheumatology and the Department of Biostatistics and Clinical Epidemiology, University of Pennsylvania (P.A.M.), and the Respiratory Therapy Area Unit and Flexible Discovery Unit, GlaxoSmithKline (J.S.), Philadelphia; Rheumazentrum, Schleswig-Holstein Mitte, Neumünster, Germany (F.M.); the Division of Pulmonary and Critical Care Medicine, Mayo Clinic, Rochester, MN (U.S.); the Vasculitis Research Unit, Department of Autoimmune Diseases, Hospital Clinic University of Barcelona, Institut d'Investigacions Biomèdiques August Pi i Sunyer, Barcelona (M.C.C.); the Respiratory Therapeutic Area, GlaxoSmithKline, Research Triangle Park, NC (S.W.Y.); and the Departments of Dermatology and Medicine, University of Utah School of Medicine, Salt Lake City (G.J.G.).

\section{REFERENCES}

1. Guillevin L, Cohen P, Gayraud M, Lhote F, Jarrousse B, Casassus P. ChurgStrauss syndrome: clinical study and longterm follow-up of 96 patients. Medicine (Baltimore) 1999;78:26-37.

2. Churg J, Strauss L. Allergic granulomatosis, allergic angiitis, and periarteritis nodosa. Am J Pathol 1951;27:277-301. 3. Jennette JC, Falk RJ, Bacon PA, et al. 2012 Revised International Chapel Hill Consensus Conference Nomenclature of Vasculitides. Arthritis Rheum 2013;65:1-11. 4. Cottin V, Bel E, Bottero P, et al. Respiratory manifestations of eosinophilic granulomatosis with polyangiitis (ChurgStrauss). Eur Respir J 2016;48:1429-41.

5. Akuthota P, Weller PF. Eosinophils and disease pathogenesis. Semin Hematol 2012;49:113-9.

6. Khoury P, Grayson PC, Klion AD. Eosinophils in vasculitis: characteristics and roles in pathogenesis. Nat Rev Rheumatol 2014;10:474-83.

7. Dunogué B, Pagnoux C, Guillevin L. Churg-Strauss syndrome: clinical symptoms, complementary investigations, prognosis and outcome, and treatment. Semin Respir Crit Care Med 2011;32:298-309.

8. Yates M, Watts RA, Bajema IM, et al.
EULAR/ERA-EDTA recommendations for the management of ANCA-associated vasculitis. Ann Rheum Dis 2016;75:1583-94. 9. Mahr A, Moosig F, Neumann T, et al. Eosinophilic granulomatosis with polyangiitis (Churg-Strauss): evolutions in classification, etiopathogenesis, assessment and management. Curr Opin Rheumatol 2014; 26:16-23.

10. Comarmond C, Pagnoux C, Khellaf M, et al. Eosinophilic granulomatosis with polyangiitis (Churg-Strauss): clinical characteristics and long-term followup of the 383 patients enrolled in the French Vasculitis Study Group cohort. Arthritis Rheum 2013;65:270-81.

11. Ribi C, Cohen P, Pagnoux C, et al. Treatment of Churg-Strauss syndrome without poor-prognosis factors: a multicenter, prospective, randomized, open-label study of seventy-two patients. Arthritis Rheum 2008;58:586-94.

12. Baldini C, Talarico R, Della Rossa A, Bombardieri S. Clinical manifestations and treatment of Churg-Strauss syndrome. Rheum Dis Clin North Am 2010; 36:527-43.

13. Samson M, Puéchal X, Devilliers H, et al. Long-term outcomes of 118 patients with eosinophilic granulomatosis with polyangiitis (Churg-Strauss syndrome) enrolled in two prospective trials. J Autoimmun 2013;43:60-9.

14. Strehl C, Bijlsma JW, de Wit M, et al. Defining conditions where long-term glucocorticoid treatment has an acceptably low level of harm to facilitate implementation of existing recommendations: viewpoints from an EULAR task force. Ann Rheum Dis 2016;75:952-7.

15. Sarnes E, Crofford L, Watson M, Dennis G, Kan H, Bass D. Incidence and US costs of corticosteroid-associated adverse events: a systematic literature review. Clin Ther 2011;33:1413-32.

16. Hellmich B, Csernok E, Gross WL. Proinflammatory cytokines and autoimmunity in Churg-Strauss syndrome. Ann N Y Acad Sci 2005;1051:121-31.

17. Haldar P, Brightling CE, Hargadon B, et al. Mepolizumab and exacerbations of refractory eosinophilic asthma. $\mathrm{N}$ Engl J Med 2009;360:973-84.

18. Pavord ID, Korn S, Howarth P, et al. Mepolizumab for severe eosinophilic asthma (DREAM): a multicentre, doubleblind, placebo-controlled trial. Lancet 2012;380:651-9. 
19. Bel EH, Wenzel SE, Thompson PJ, et al. Oral glucocorticoid-sparing effect of mepolizumab in eosinophilic asthma. N Engl J Med 2014;371:1189-97.

20. Ortega HG, Liu MC, Pavord ID, et al. Mepolizumab treatment in patients with severe eosinophilic asthma. N Engl J Med 2014;371:1198-207.

21. Nair P, Pizzichini MMM, Kjarsgaard $\mathrm{M}$, et al. Mepolizumab for prednisonedependent asthma with sputum eosinophilia. N Engl J Med 2009;360:985-93.

22. Kahn JE, Grandpeix-Guyodo C, Marroun I, et al. Sustained response to mepolizumab in refractory Churg-Strauss syndrome. J Allergy Clin Immunol 2010;125: 267-70.

23. Kim S, Marigowda G, Oren E, Israel E, Wechsler ME. Mepolizumab as a steroidsparing treatment option in patients with Churg-Strauss syndrome. J Allergy Clin Immunol 2010;125:1336-43.

24. Moosig F, Gross WL, Herrmann K, Bremer JP, Hellmich B. Targeting interleukin-5 in refractory and relapsing Churg-Strauss syndrome. Ann Intern Med 2011;155:341-3.
25. Groh M, Pagnoux C, Baldini C, et al. Eosinophilic granulomatosis with poly angiitis (Churg-Strauss) (EGPA) Consensus Task Force recommendations for evaluation and management. Eur J Intern Med 2015;26:545-53.

26. Mukhtyar C, Lee R, Brown D, et al. Modification and validation of the Birmingham Vasculitis Activity Score (version 3). Ann Rheum Dis 2009;68:1827-32. 27. Hellmich B, Flossmann O, Gross WL, et al. EULAR recommendations for conducting clinical studies and/or clinical trials in systemic vasculitis: focus on antineutrophil cytoplasm antibody-associated vasculitis. Ann Rheum Dis 2007;66: 605-17.

28. Cloutier MM, Schatz M, Castro M, et al. Asthma outcomes: composite scores of asthma control. J Allergy Clin Immunol 2012;129:Suppl:S24-S33.

29. Juniper EF, O'Byrne PM, Guyatt GH, Ferrie PJ, King DR. Development and validation of a questionnaire to measure asthma control. Eur Respir J 1999;14:9027.

30. Rothenberg ME, Klion AD, Roufosse
FE, et al. Treatment of patients with the hypereosinophilic syndrome with mepolizumab. N Engl J Med 2008;358:1215-28.

31. Annapureddy N, Elsallabi O, Baker J, Sreih AG. Patient-reported outcomes in ANCA-associated vasculitis: a comparison between Birmingham Vasculitis Activity Score and routine assessment of patient index data 3. Clin Rheumatol 2016;35:395-400.

32. Dejaco C, Oppl B, Monach P, et al. Serum biomarkers in patients with relapsing eosinophilic granulomatosis with polyangiitis (Churg-Strauss). PLoS One 2015;10(3): e0121737.

33. Grayson PC, Monach PA, Pagnoux C, et al. Value of commonly measured laboratory tests as biomarkers of disease activity and predictors of relapse in eosinophilic granulomatosis with polyangiitis. Rheumatology (Oxford) 2015;54:1351-9. 34. Mohammad AJ, Hot A, Arndt F, et al. Rituximab for the treatment of eosinophilic granulomatosis with polyangiitis (Churg-Strauss). Ann Rheum Dis 2016;75: 396-401.

Copyright $\odot 2017$ Massachusetts Medical Society. 